\title{
Grasp: Design and Preliminary Testing of an Open-Source, 3D Printable Bionic Hand
}

\author{
Xavier Cano-Ferrer ${ }^{1}$ and Sergio Gómez-González ${ }^{1,2}$
}

\begin{abstract}
We present the design and performance evaluation of a 3D printable bionic hand developed to be manufactured, assembled and programmed in the simplest way by the user. This prosthetic device can be controlled by surface electromyography (EMG) performing the six most important types of grasp to achieve the activities of daily living (ADL's). Our design represents an affordable option with competitive features when compared to the existing commercial and open source devices.
\end{abstract}

\section{INTRODUCTION}

There are 3 millions of upper limb amputees worldwide, and this represents a minority of $5 \%$ of the total number of amputees [1]. This is one of the reasons as to why the production costs of an advanced bionic limb are so elevated. This impacts on the acquisition prices, setting them between 25.000 and $75.000 €[2]$. The main motivation of the present work has been to create a design of a bionic hand with 6 degrees of freedom (DOF), which can be $3 \mathrm{D}$ printed with competitive features in relation to the best commercial models available in the market (Figure 1).

The main objectives of the present work can be summarised as follows: (1) the bionic hand must be able to reproduce the six most important types of grips required in order to carry out activities of daily life (ADL's) [2]; (2) all the components: electronics and actuators should be included into the palm of the hand to reduce the possible space occupied in the forearm; (3) the hand mass should be less than $400 \mathrm{~g}$ [2], which represents the weight critical for the user to be considered too heavy; (4) the bionic hand should be easily assembled and programmed by the user once the open source files have been downloaded; (5) the total cost of the hand must be below $500 €$ including all components.

\footnotetext{
1 Department of Engineering Presentation, Polytechnic University of Catalonia (UPC)

${ }^{2}$ Research Group of Interacting Surfaces in Bioengineering and Materials Science (InSup), Polytechnic University of Catalonia (UPC)

Corresponding authors: xaviercanoferrer@gmail.com and sergi.gomez-gonzalez@upc.edu
}

Finally, the greatest challenge is to achieve all these objectives and at the same time be competitive with respect to existing commercial projects.

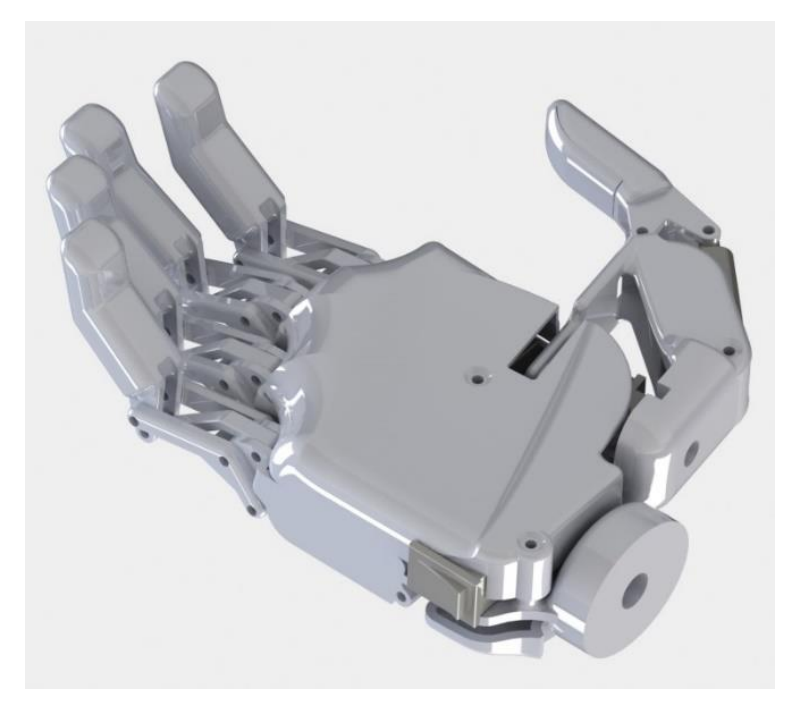

Figure 1: Grasp bionic hand.

\section{HARDWARE DESIGN}

a. Mechanical design

The dimensions of our bionic device are close to the average anthropometric values of the actual human hand, as reported in Mosquera et al. [3] (Figure 2).

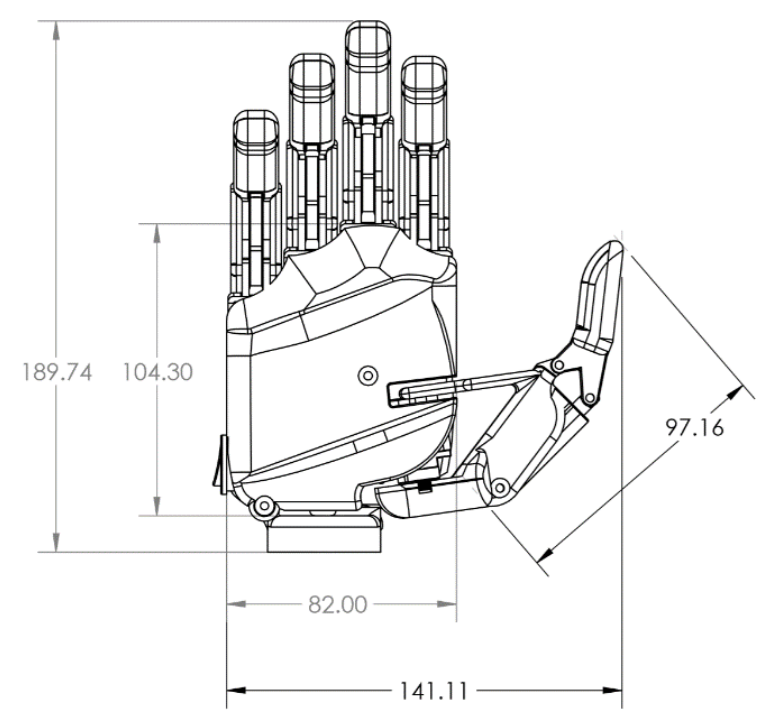

Figure 2: Dimensions of the prosthetic device. 
The hand has six degrees of actuation: flexionextension of each finger and oppositionretroposition of the thumb. The workspace of the hand has been simulated extracting the trajectory values from the CAD software Solidworks ${ }^{\circledR}$ kinematics simulation and plotted in Matlab® (Figure 3). With the same simulation data, the estimated values of flexion-extension speed and opposition-retroposition of the thumb are $123 \%$ and $600 \%$, respectively. The first value comes from a linear displacement made by the actuator which is $15 \mathrm{~mm}$ at the speed of $15 \mathrm{~mm} / \mathrm{s}$ with a flexion of $123 \%$ from the initial angle. The angular velocity of the thumb opposition is the value of the rotary servo motor rotation speed.

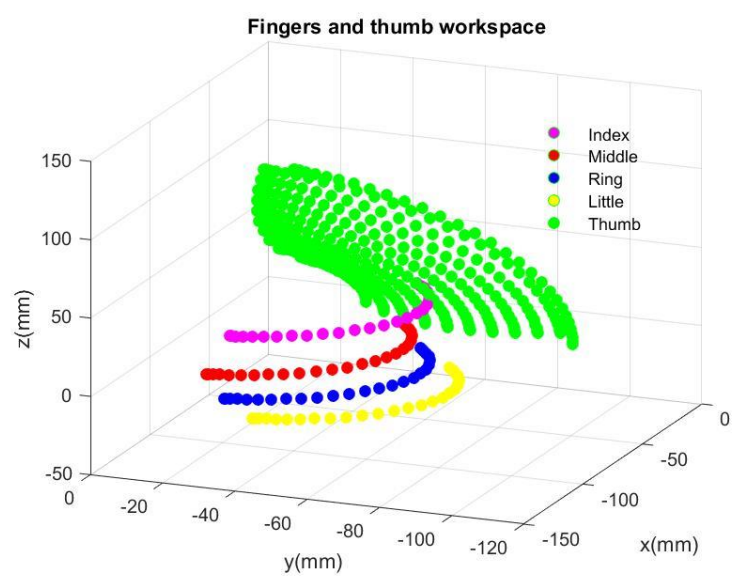

Figure 3: Simulated workspace of the hand.

The flexion-extension finger movements are actuated by five DC linear servo motor Actuonix PQ12R 63:1 gear ratio. The oppositionretroposition movement of the thumb is actuated by a DC rotary servo motor Turnigy ${ }^{\circledR}$ MG90S. The maximum fingertip forces for the flexion-extension movement with the finger in the extended position have been calculated for both types of mechanical systems: the finger and the thumb systems. Using a Matlab® script, the increase of an external load applied (W) sets the relationship between the external load and the actuator force (F) (Figure 4). The stall force of the linear actuator provided by the manufacturer $(45 \mathrm{~N})$ allows the calculation of the maximum fingertip forces as shown below in equations 1 and 2 :

$$
\begin{aligned}
& W_{\text {finger }}=\frac{1}{7.74} F_{\text {maxactuator }}[N] \\
& W_{\text {thumb }}=\frac{1}{2.73} F_{\text {maxactuator }}[N]
\end{aligned}
$$

The estimated value of the maximum fingertip force achieved by the finger and the thumb mechanical systems are $5.81 \mathrm{~N}$ and $16.49 \mathrm{~N}$ respectively.

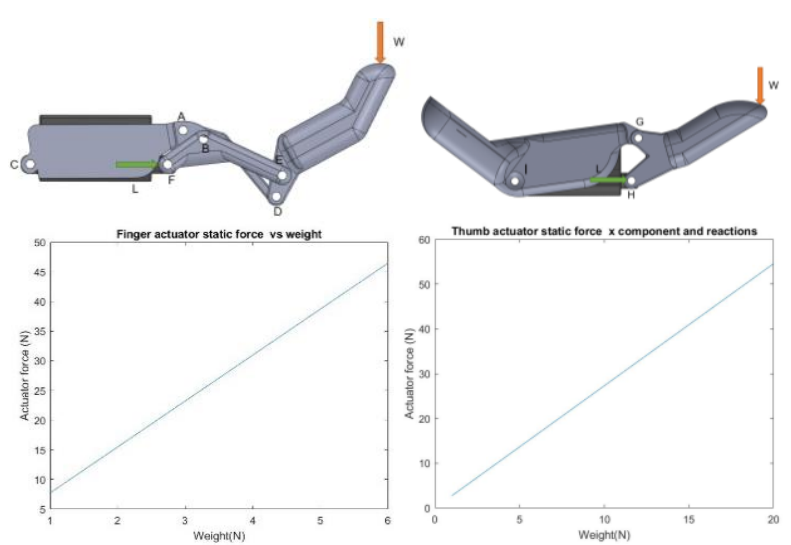

Figure 4: Relationship between the actuator force and the external load applied on the tip of the finger.

\section{b. Electrical design}

The microcontroller selected to control the servo motors is the Arduino Nano, a highly used system in open source projects by its small dimensions. Grasp Bionic Hand requires at least 6 Digital I/O with pulse width modulation (PWM) to control the servo motors and one analogue pin for the input muscle signal. The surface EMG sensor used is the Advancer Technologies ${ }^{\circledR}$ Myoware. The design requires any pushbutton module. To estimate the maximum current of the whole system, one needs to consider all the maximum current values for each electrical component, as shown in equation 3 :

$$
I_{\text {max }}=\sum_{i=1}^{n} I_{i}
$$

The value of the maximum current is $2650 \mathrm{~mA}$ considering that all the actuators work at stall current (Figure 5). To achieve these high currents, we employ Lithium Polymer (Li-Po) batteries. LiPo batteries can supply these high DC current while their voltage of $7.4 \mathrm{~V}$ is the closest to actuator voltage $(6 \mathrm{~V})$. To overcome this voltage difference, we make use of a Pololu ${ }^{\circledR}$ step down voltage regulator $\mathrm{D} 24 \mathrm{~V} 25 \mathrm{~F} 6$ with maximum continuous current value of $2.5 \mathrm{~A}$ and efficiency over $95 \%$. The selected ON/OFF switch button has a maximum DC current of $10 \mathrm{~A}$ and therefore is suitable for our system. 


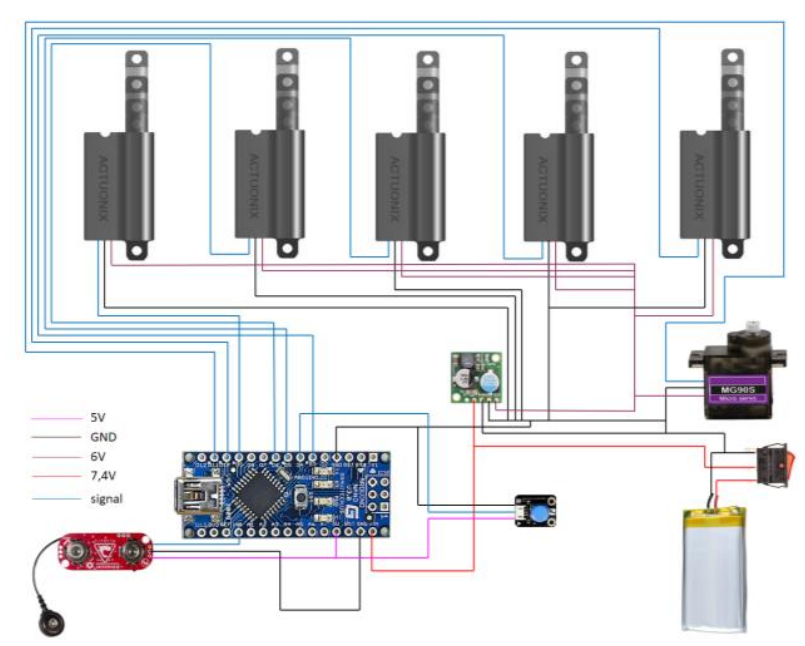

Figure 5: Electrical schematic.

\section{SOFTWARE DESIGN}

The different types of grasping patterns can be achieved by changing across the different grasp functions. Each grasp function is called by the main loop function when an external pushbutton located in the dorsal cover is pressed (Figure 6).

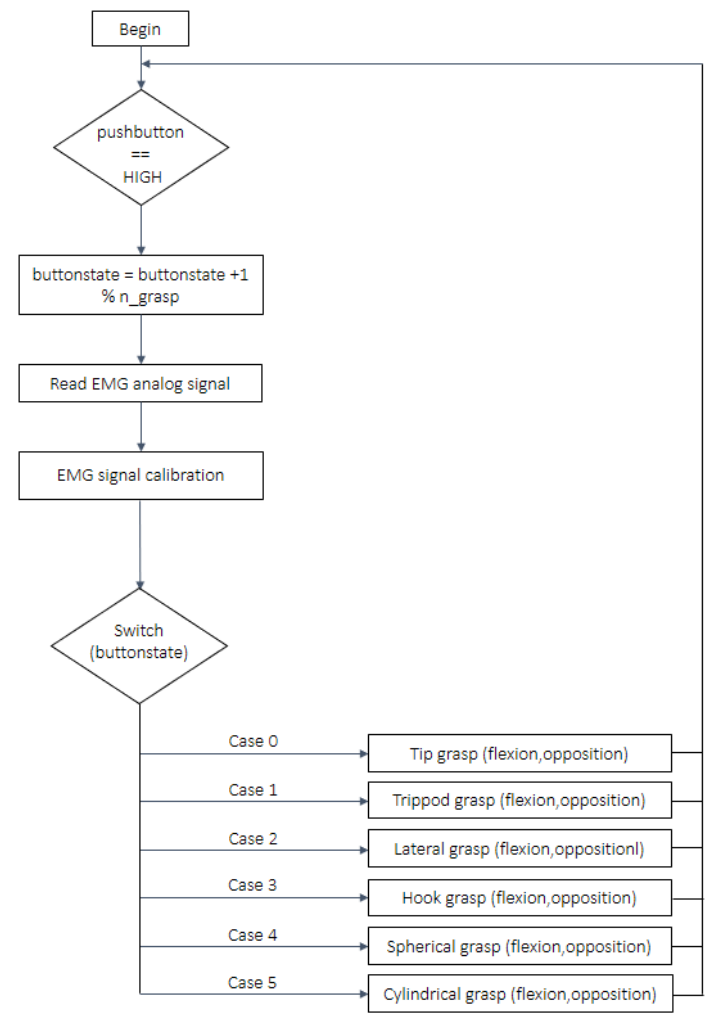

Figure 6: Main function for the hand control.

The hand is controlled by surface EMG using a linear control. The maximum value of the EMG signal has interpersonal variability (max_subject). For this reason, this value must be introduced at the beginning of the program as a constant value. The output response will remain in the range of values set by the maximum value of the subject signal. The angle given to the actuator by the servo.write() function of the servo.h library is processed from the EMG input signal with the equation 4:

$$
\varphi=\frac{-107}{\text { max_subject }} \cdot E M G+145
$$

\section{RESULTS AND PROTOTYPE EVALUATION}

In order to evaluate our bionic hand prototype, the hand was printed in PLA 3D850 and manually assembled. We have compared its performance to the data obtained from our simulations and with other 6 DOF commercial and open source models available. The prototype of the hand has been evaluated using three main experiments: qualitative object grasping, finger actuation speeds and fingertip isometric forces. In the first experiment, we demonstrate that the hand is capable to perform the six types of grasping patterns: lateral, palmar, tip, hook, spherical and cylindrical (Figure 7).

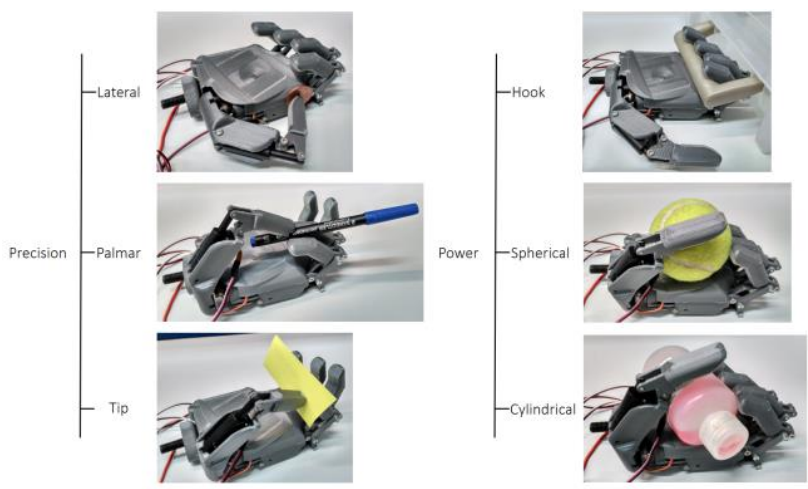

Figure 7: The bionic device performing the most important six types of grasp.

In the second experiment, using $\mathrm{N}=3$ samples and image processing with Kinovea software, we obtained maximum flexion-extension speed values of $72 \pm 16 \%$ and $196 \pm 60 \%$ for the finger and thumb, respectively (Figure 8).

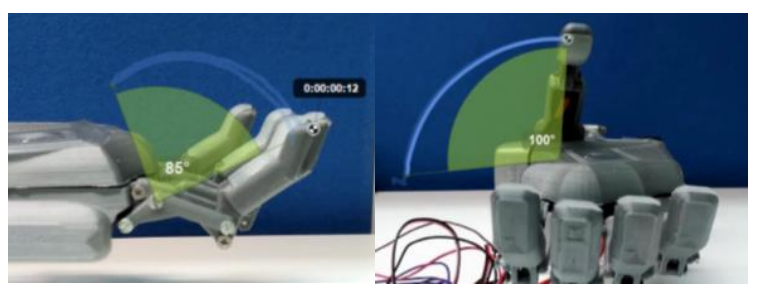

Figure 8: Kinematics evaluation for the finger and the thumb, respectively. 
In the third experiment we have evaluated the fingertip forces using $\mathrm{N}=5$ samples and the FSR 402 force sensing resistor. In both types of fingers, by applying an isometric force against the sensor we obtained fingertip force values of $6.82 \pm 0.14 \mathrm{~N}$ and $14.50 \pm 0.21 \mathrm{~N}$, for the finger and the thumb, respectively (Figure 9).

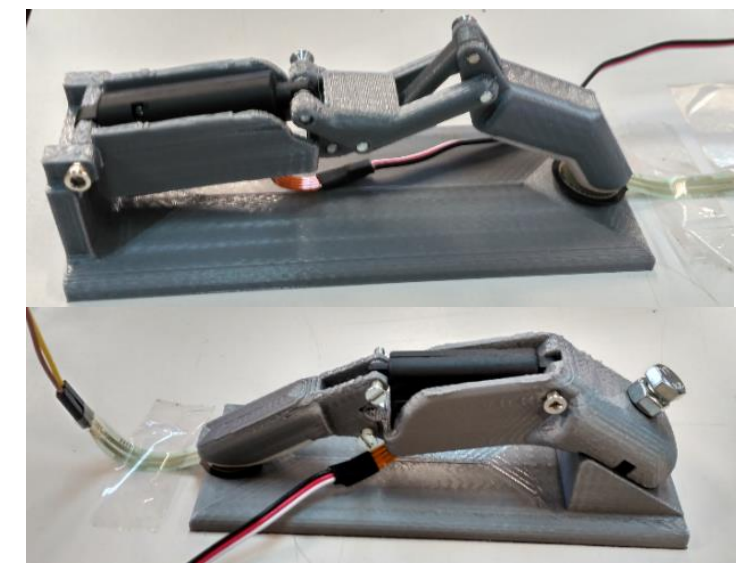

Figure 9: Fingertip forces setup with the thumb in extended isometric position applying force against the sensor.

The final mass of the prosthetic device is $330 \mathrm{~g}$, value under the average mass of the actual human hand and under the critical value of $400 \mathrm{~g}$ [2].

The final step of the experimental procedure was the EMG control integrating all the electronic and mechanical components described previously (Figure 10). The EMG signal controls the flexionextension movement of the fingers involved on the grasp pattern and the pushbutton switches from one type of grasp to the next one.

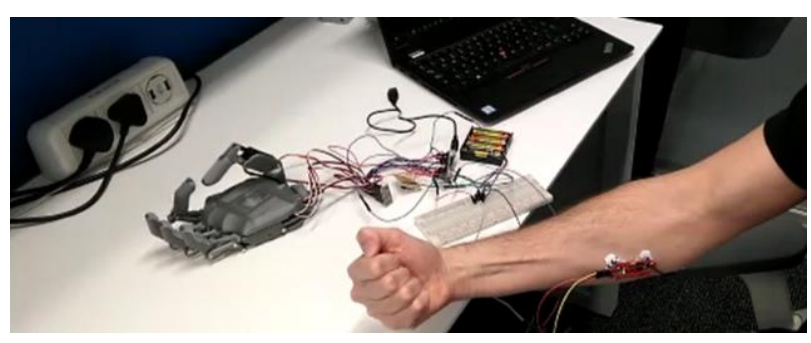

Figure 10: EMG control test of the hand using a pushbutton to switch the type of grasp [13].

\section{DISCUSSION}

The maximum speed achieved in the commercial prosthetic devices is $110.60 \% \mathrm{~s}$ and this is achieved by the i-Limb [4]. The highest value on fingertip force is $16.11 \mathrm{~N}$ and this is achieved by the Bebionic [4]. The open source hands Tact and Dextrus have high average speed values: 249.80 and $175.4 \%$ (Figure 11) but, with less fingertip forces: 4.21 and $1.71 \mathrm{~N}$, respectively (Figure 12). With the values obtained with our novel hand Grasp (Table 1), the device presented in this work represents an affordable (477.35 $€)$ and competitive bionic hand.

Table 1: Summary of Grasp hand features.

\begin{tabular}{ll} 
Flexion-extension speed $(\%)$ & 71.96 \\
\hline Opposition speed $(\%)$ & 196.10 \\
\hline Finger fingertip force $(\mathbf{N})$ & 6.82 \\
\hline Thumb fingertip force $(\mathbf{N})$ & 16.50 \\
\hline Mass $(\mathbf{g})$ & 335 \\
\hline DOF & 6 \\
\hline Size $(\mathbf{m m})$ & $190 \times 141 \times 53$ \\
\hline Cost $(\boldsymbol{€})$ & 477.35 \\
\hline
\end{tabular}

Although the features of our 6 DOF bionic device are substantial, one needs to always take into consideration that the values of fingertip forces of the actual human hand can be around $30 \mathrm{~N}[5,6]$ and the flexion-extension speed values of up to 384 $\%$ [7]. Thus, 3D printed open source prosthetic devices are still far away from the 25 DOF actual human hand [8].

Grasp Bionic Hand has been published under a Creative Commons License-Attribution-Non Commercial in Hackaday [11] and Thingiverse [12]. 


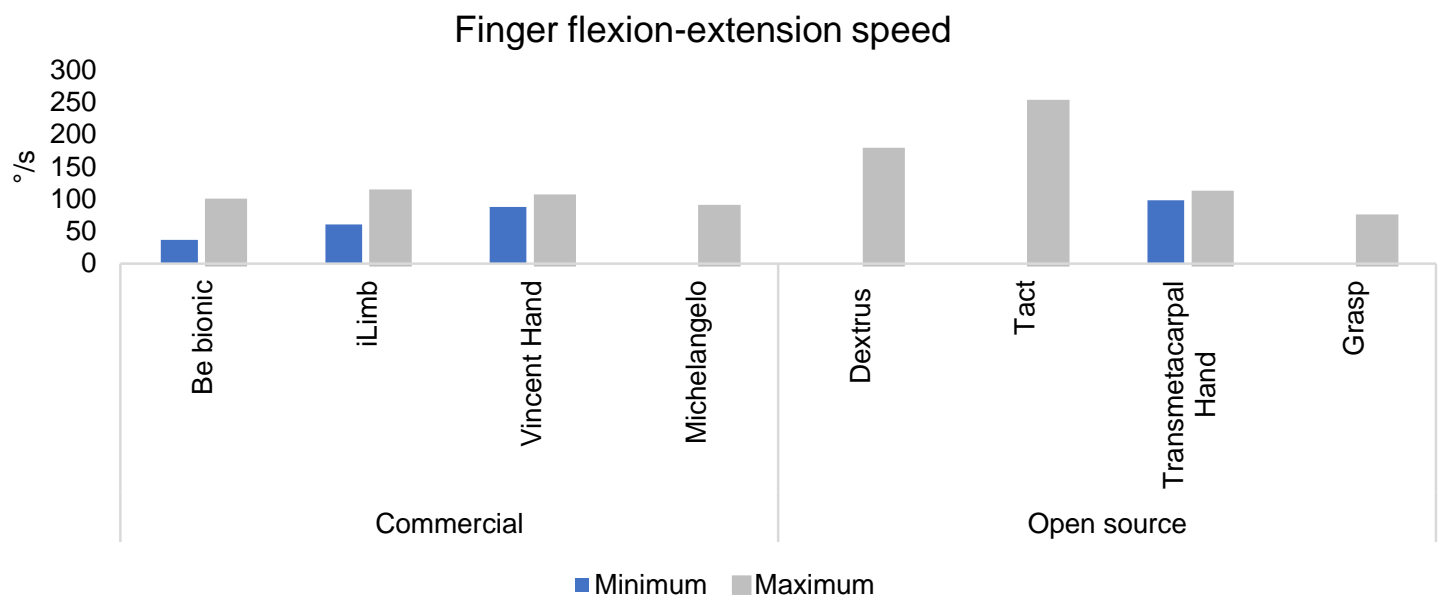

Figure 11: Flexion-extension speed of Grasp hand compared with other commercial and open source hands [4, 9, 10].

Fingertip force

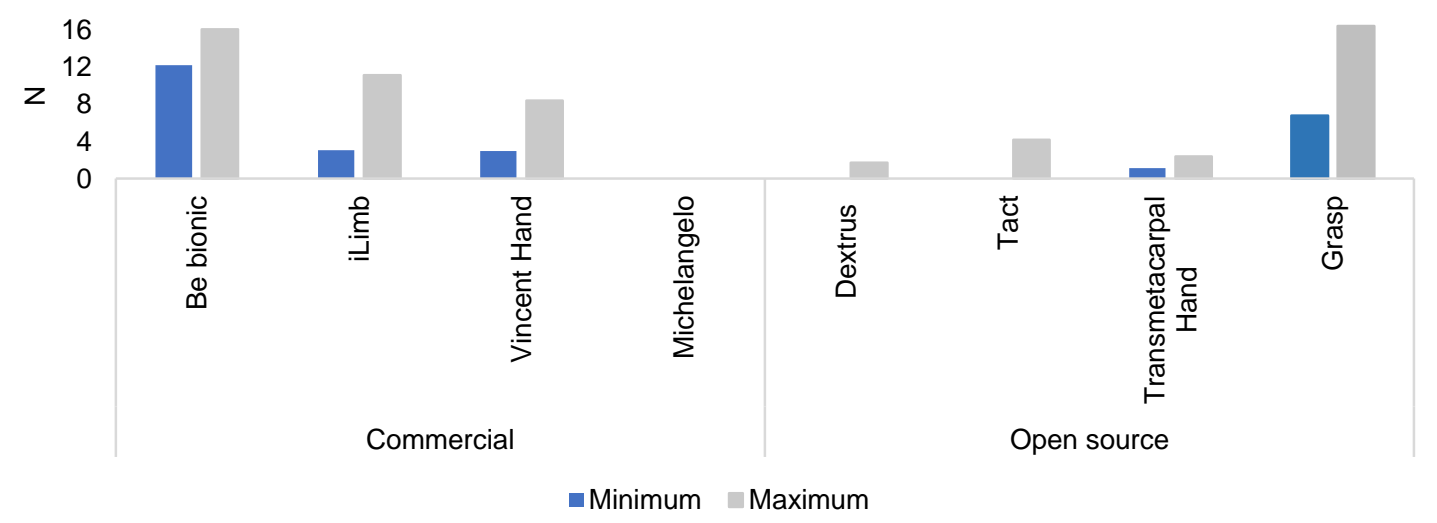

Figure 12: Fingertip forces of Grasp hand compared with other commercial and open source hands [4, 9, 10].

\section{REFERENCES}

[1] M. L. Blanc, "“Give Hope - Give a Hand" - The LN-4," 2008.

[2] J. t. Kate, G. Smith and P. Breedveld, "3D printed upper limb prostheses: a review.," Disability and rehabilitation: Assistive technology, vol. 12, no. 3, pp. 300-314, 2017.

[3] L. Mosquera Camelo and V. Guedez, "Estudio biomecánico de la mano durante el agarre de herramientas manuales Datos antropométricos preliminares.," in 3rd International Conference on Occupational Risk and Prevention, 2004.

[4] J. T. Belter, J. L. Segil, A. M. Dollar and R. F. Weir, "Mechanical design and performance specifications of antrophometric prosthetic hands: A review," Journal of Rehabilitation
Research \& Development (JRRD), vol. 50, no. 5, pp. 599-618, 2013.

[5] K. Shima, Y. Tamura, T. Tsuji, A. Kandori, M. Yokoe and S. Sakoda, "Estimation of Human Finger Tapping Forces Based on a FingerpadStiffness Model," in 31st Annual International Conference of the IEEE EMBS, Minneapolis, 2009.

[6] P. Wattanasiri, P. Tangpornprasert and V. Chanyaphan, "Design of Multi-Grip Patterns Prosthetic Hand with Single Actuator," IEEE Transactions on Neural Systems and Rehabilitation Engineering, vol. 26, no. 7, pp. 1188 - 1198, 2018.

[7] D. van der Riet, R. Stopforth, G. Bright and O. Diegel, "THE LOW COST DESIGN OF A 3D 
PRINTED

MULTI-FINGERED

MYOELECTRIC PROSTHETIC HAND," in

Mechatronics: Principles, Technologies and

Applications, Nova Science Publishers, 2015, pp. 86-116.

[8] E. Peña-Pitarch, N. Ticó Farguera and J. (. Yang, "Virtual human hand: model and kinematics," Computer Methods in Biomechanics and Biomedical Engineering, pp. 1-12, 2012.

[9] P. Slade, A. Akhtar, M. Nguyen and T. Bretl, "Tact: Design and Performance of an OpenSource, Affordable, Myoelectric Prosthetic Hand," 2015.

[10] R. Mio, B. Villegas, L. Ccorimanya, K. M. Flores, G. Salazar and D. Elías, "Development and Assessment of a Powered 3D-printed Prosthetic Hand for Transmetacarpal Amputees," in International Conference on Control, Automation and Robotics, 2017.

[11] “https://hackaday.io/project/163935-graspbionic-hand," [Online].

[12] "https://www.thingiverse.com/thing:2736588," [Online].

[13] "https://www.youtube.com/watch?v=ftML2nO W1Do" [Online]. 